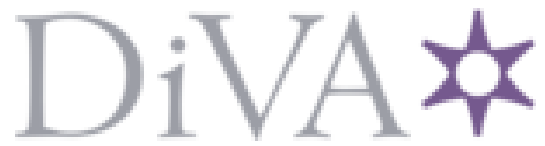

http://www.diva-portal.org

Postprint

This is the accepted version of a paper presented at 59th IEEE Conference on Decision and Control, Jeju Island, Republic of Korea, December 14-18, 2020.

Citation for the original published paper:

Chen, F., Dimarogonas, D V. (2020)

Further Results on Leader-follower Multi-agent Formation Control with Prescribed Performance Guarantees

In:

N.B. When citing this work, cite the original published paper.

Permanent link to this version:

http://urn.kb.se/resolve?urn=urn:nbn:se:kth:diva-286305 


\title{
Further Results on Leader-follower Multi-agent Formation Control with Prescribed Performance Guarantees
}

\author{
Fei Chen and Dimos V. Dimarogonas
}

\begin{abstract}
Distributed formation control for leader-follower multi-agent systems under prescribed performance guarantees is addressed in this paper. Leader-follower is meant in the sense that a group of agents with external inputs are selected as leaders in order to drive the group of followers in a way that the entire system can achieve the target relative position-based formation within certain prescribed performance transient bounds. In previous work, we have proposed a distributed control law for tree graphs to achieve consensus within certain prescribed transient performance when the decay rate of the performance functions is within a sufficient bound. In this paper, we further discuss the general graphs with cycles. Some necessary conditions on the graph topology are proposed in order to achieve the target formation while satisfying the prescribed performance bounds. We also discuss the roles of the cycles for the convergence benefits in this leader-follower framework. Finally, we illustrate the results with the simulation examples.
\end{abstract}

\section{INTRODUCTION}

The class of relative position-based formation control [11] is specified by desired relative positions between neighboring agents, which plays an important role in achieving or maintaining desired geometrical patterns via the cooperation of multiple robots. In [11], the authors summarise the first and second-order relative position-based formation protocols that are extended from the first-order [12] and second-order [13] consensus protocols, respectively.

In this work, we study relative position-based formation control in a leader-follower framework, that is, one or more agents are selected as leaders with external inputs in addition to the first-order formation protocol. The remaining agents are followers only obeying the first-order formation protocol. Related research that has been done in the leaderfollower framework mainly focus on controllability of leaderfollower multi-agent systems [7] and also leader selection problems [14], [5] to achieve optimal performance.

Prescribed performance control (PPC) was originally proposed in [1] to prescribe the evolution of system output or the tracking error within some predefined region. When it comes to multi-agent systems, PPC for multi-agent average consensus with single integrator dynamics is presented in [8]. Compared with classical results of consensus or formation control for multi-agent systems, applying PPC for multiagent systems can achieve additional transient behavior besides the consensus or formation purpose. Funnel control,

This work was supported by the ERC Consolidator Grant LEAFHOUND, the EU H2020 Co4Robots Project, the Swedish Research Council (VR) and the Knut och Alice Wallenberg Foundation (KAW).

Fei Chen and Dimos V. Dimarogonas are with the Division of Decision and Control Systems, KTH Royal Institute of Technology, SE-100 44 Stockholm, Sweden $\{$ fchen, dimos $\}$ ekth. se which uses a similar idea as PPC was introduced in [6] for reference tracking.

In such a leader-follower framework, we aim to design the leaders only such that the entire system achieves a relative position-based formation within certain performance bounds. We apply a PPC law only to the leaders while the followers will just follow the leaders by obeying the first-order formation protocol without any further control and knowledge of the prescribed team bounds. Compared with [9], in which the multi-agent system only has one leader that is treated as a reference for the followers, we focus on a more general framework in the sense that we can have more than one leader and the leaders are designed to steer the leaderfollower multi-agent system achieving the target formation within the prescribed performance bounds. The combination of uncertain topologies, leader amount and leader positions makes the problem tough to solve. Moreover, the leader can only communicate with its neighboring agents. Preliminary results of first and second-order consensus for leaderfollower multi-agent systems with prescribed performance guarantees have been presented in [2], [3], respectively. In this paper, we extend our previous results to more general graphs with cycles and discuss how the cycles will benefit the convergence results. The extension of general graphs with cycles has more practical applications compared with our previous results for tree graphs and offers a complete theory for undirected graphs. The challenge of general graphs with cycles is that we cannot utilize the positive definiteness of the edge Laplacian for convergence analysis directly, thus we need to partition the graph into a spanning tree and the remaining edges that complete the cycles. We also derive necessary conditions on the graph topologies under which we can design the leaders to achieve the target formation within certain prescribed performance bounds. This introduces for the first time criteria on how to choose the leader amounts and leader positions to meet the target formation while satisfying the prescribed transient performance behavior. The contributions of the paper can be summarized as: i) within a general leader-follower framework, a distributed control law is proposed for general graphs with cycles when the decay rate of the performance functions is within a sufficient bound and the convergence benefits of the cycles are discussed; ii) necessary conditions on the graph topologies are proposed such that under these conditions we can further design the leaders to achieve the target formation with prescribed performance guarantees.

The rest of the paper is organized as follows. In Section II, preliminary knowledge is introduced and the problem 
is formulated, while Section III presents the result of relative position-based formation control for leader-follower multi-agent systems that contain cycles within prescribed performance bounds. Further necessary conditions on the graph topologies are discussed in Section IV. The results are verified by some simulations and examples in Section V. Section VI includes conclusions and future work.

\section{PRELIMINARIES AND PROBLEM STATEMENT}

\section{A. Graph Theory}

An undirected graph [10] is defined as $\mathcal{G}=(\mathcal{V}, \mathcal{E})$ with the vertices set $\mathcal{V}=\{1,2, \ldots, n\}$ and the edges set $\mathcal{E}=$ $\left\{(i, j) \in \mathcal{V} \times \mathcal{V} \mid j \in \mathcal{N}_{i}\right\}$ indexed by $e_{1}, \ldots, e_{m}$. Here, $m=$ $|\mathcal{E}|$ is the number of edges and $\mathcal{N}_{i}$ denotes the neighbourhood of agent $i$ such that agent $j \in \mathcal{N}_{i}$ can communicate with $i$. A path is a sequence of edges connecting two distinct vertices. A graph is connected if there exists a path between any pair of vertices. By assigning an orientation to each edge of $\mathcal{G}$ the incidence matrix $D=D(\mathcal{G})=\left[d_{i j}\right] \in \mathbb{R}^{n \times m}$ is defined. The rows of $D$ are indexed by the vertices and the columns are indexed by the edges with $d_{i j}=1$ if the vertex $i$ is the head of the edge $(i, j), d_{i j}=-1$ if the vertex $i$ is the tail of the edge $(i, j)$ and $d_{i j}=0$ otherwise. The graph Laplacian of $\mathcal{G}$ is described as $L=D D^{T}$. In addition, $L_{e}=D^{T} D$ is the so called edge Laplacian [15].

\section{B. System Description}

In this work, we consider a multi-agent system with vertices $\mathcal{V}=\{1,2, \ldots, n\}$. Suppose that the first $n_{f}$ agents are followers while the last $n_{l}$ agents are leaders with $\mathcal{V}_{F}=$ $\left\{1,2, \ldots, n_{f}\right\}, \mathcal{V}_{L}=\left\{n_{f}+1, n_{f}+2, \ldots, n_{f}+n_{l}\right\}$ and $n=n_{f}+n_{l}$. Here and later on, the subscript " $F$ ", " $L$ " stands for follower and leader vertices set, respectively. We also denote the follower-follower edge set as $\mathcal{E}_{F F}=\left\{e_{k} \mid\right.$ $\left.1 \leq k \leq m, k \in \mathbb{Z}, e_{k}=(i, j), i, j \in \mathcal{V}_{F}\right\}$.

Let $p_{i} \in \mathbb{R}$ be the position of agent $i$, where we only consider the one dimensional case, without loss of generality. Specifically, the results can be extended to higher dimensions with appropriate use of the Kronecker product. The target relative position-based formation is described as follows:

$$
\mathcal{F}:=\left\{p \mid p_{i}-p_{j}=p_{i j}^{d e s},(i, j) \in \mathcal{E}\right\},
$$

where $p_{i j}^{\text {des }}:=p_{i}^{\text {des }}-p_{j}^{\text {des }},(i, j) \in \mathcal{E}$ is the desired relative position between agent $i$ and agent $j$, which is constant and denoted as the difference between the absolute desired positions $p_{i}^{\text {des }}, p_{j}^{\text {des }} \in \mathbb{R}$. Here, $p_{i j}^{\text {des }}$ is only needed to be known and $p_{i}^{d e s}, p_{j}^{d e s}$ are defined with respect to an arbitrary reference frame and do not need to be known. The state evolution of each follower $i \in \mathcal{V}_{F}$ is governed by the firstorder formation protocol:

$$
\dot{p}_{i}=-\sum_{j \in \mathcal{N}_{i}}\left(p_{i}-p_{j}-p_{i j}^{d e s}\right),
$$

while for the leader $i \in \mathcal{V}_{L}$, it obeys the following first-order formation protocol with an assigned external input $u_{i} \in \mathbb{R}$ :

$$
\dot{p}_{i}=-\sum_{j \in \mathcal{N}_{i}}\left(p_{i}-p_{j}-p_{i j}^{\text {des }}\right)+u_{i} .
$$

We denote $p=\left[p_{1}, \ldots, p_{n}\right]^{T}, p^{\text {des }}=\left[p_{1}^{\text {des }}, \ldots, p_{n}^{\text {des }}\right]^{T} \in$ $\mathbb{R}^{n}$ as the respective stack vector of absolute positions and target positions and $u=\left[u_{n_{f}+1}, \ldots, u_{n_{f}+n_{l}}\right]^{T} \in \mathbb{R}^{n_{l}}$ is the control input vector that contains the external inputs of leader agents in (3). Denote $\bar{p}=\left[\bar{p}_{1}, \ldots, \bar{p}_{m}\right]^{T}, \bar{p}^{\text {des }}=$ $\left[\bar{p}_{1}^{\text {des }}, \ldots, \bar{p}_{m}^{\text {des }}\right]^{T} \in \mathbb{R}^{m}$ as the respective stack vector of relative positions and target relative positions between the pair of communication agents for the edge $(i, j)=e_{k} \in \mathcal{E}$, where $\bar{p}_{k} \triangleq p_{i j}=p_{i}-p_{j}, \bar{p}_{k}^{\text {des }} \triangleq p_{i j}^{\text {des }}=p_{i}^{\text {des }}-p_{j}^{\text {des }}, k=$ $1,2, \ldots, m$. It can be then verified that $L p=D \bar{p}$ and $\bar{p}=D^{T} p$. In addition, if $\bar{p}=0$, we have that $L p=0$. Similarly, it holds that $L p^{\text {des }}=D \bar{p}^{\text {des }}, \bar{p}^{\text {des }}=D^{T} p^{\text {des }}$.

By stacking (2) and (3), the dynamics of the leaderfollower multi-agent system is rewritten as:

$$
\Sigma: \dot{p}=-L\left(p-p^{\text {des }}\right)+B u,
$$

where $L$ is the graph Laplacian and $B=\left[\begin{array}{c}0_{n_{f}} \times n_{l} \\ I_{n_{l}}\end{array}\right]$.

In the sequel, we denote $x=p-p^{\text {des }}=\left[x_{1}, \ldots, x_{n}\right]^{T}$ as the shifted absolute position vector with respect to $p^{\text {des }}$. Accordingly, $\bar{x}=\bar{p}-\bar{p}^{\text {des }}=\left[\bar{x}_{1}, \ldots, \bar{x}_{m}\right]^{T}$ is denoted as the shifted relative position vector with respect to $\bar{p}^{\text {des }}$.

\section{Prescribed Performance Control}

The aim of PPC is to prescribe the evolution of the relative position $\bar{p}_{i}(t)$ within some predefined region described as

$$
\bar{p}_{i}^{\text {des }}-\rho_{\bar{x}_{i}}(t)<\bar{p}_{i}(t)<\bar{p}_{i}^{\text {des }}+\rho_{\bar{x}_{i}}(t),
$$

or equivalently, to prescribe the evolution of the shifted relative position $\bar{x}_{i}(t)$ within

$$
-\rho_{\bar{x}_{i}}(t)<\bar{x}_{i}(t)<\rho_{\bar{x}_{i}}(t) .
$$

Here $\rho_{\bar{x}_{i}}(t): \mathbb{R}_{+} \rightarrow \mathbb{R}_{+} \backslash\{0\}, i=1,2, \ldots, m$ are positive, smooth and strictly decreasing performance functions that introduce the predefined bounds for the shifted relative positions. One example choice is

$$
\rho_{\bar{x}_{i}}(t)=\left(\rho_{\bar{x}_{i 0}}-\rho_{\bar{x}_{i \infty}}\right) e^{-l_{\bar{x}_{i}} t}+\rho_{\bar{x}_{i \infty}} .
$$

with $\rho_{\bar{x}_{i 0}}, \rho_{\bar{x}_{i \infty}}$ and $l_{\bar{x}_{i}}$ positive parameters and $\rho_{\bar{x}_{i \infty}}=$ $\lim _{t \rightarrow \infty} \rho_{\bar{x}_{i}}(t)$ represents the tracking error at steady state.

Normalizing $\bar{x}_{i}(t)$ with respect to the performance function $\rho_{\bar{x}_{i}}(t)$, we define the modulated error as $\hat{\bar{x}}_{i}(t)=\frac{\bar{x}_{i}(t)}{\rho_{\bar{x}_{i}}(t)}$ and the corresponding prescribed performance region as $\mathcal{D}_{\bar{x}_{i}} \triangleq\left\{\hat{\bar{x}}_{i}: \hat{\bar{x}}_{i} \in(-1,1)\right\}$. Then the modulated error is transformed through a transformed function $T_{\bar{x}_{i}}$ that defines the smooth and strictly increasing mapping $T_{\bar{x}_{i}}: \mathcal{D}_{\bar{x}_{i}} \rightarrow \mathbb{R}$, $T_{\bar{x}_{i}}(0)=0$. One example choice is

$$
T_{\bar{x}_{i}}\left(\hat{\bar{x}}_{i}\right)=\ln \left(\frac{1+\hat{\bar{x}}_{i}}{1-\hat{\bar{x}}_{i}}\right) .
$$

The transformed error is then defined as $\varepsilon_{\bar{x}_{i}}\left(\hat{\bar{x}}_{i}\right)=T_{\bar{x}_{i}}\left(\hat{\bar{x}}_{i}\right)$ It can be verified that if the transformed error $\varepsilon_{\bar{x}_{i}}\left(\hat{\bar{x}}_{i}\right)$ is bounded, then the modulated error $\hat{\bar{x}}_{i}$ is constrained within the region $\mathcal{D}_{\bar{x}_{i}}$. This also implies the error $\bar{x}_{i}$ evolves within the predefined performance bounds (6). Differentiating $\varepsilon_{\bar{x}_{i}}\left(\hat{\bar{x}}_{i}\right)$ with respect to time, we derive

$$
\dot{\varepsilon}_{\bar{x}_{i}}\left(\hat{\bar{x}}_{i}\right)=\mathcal{J}_{T_{\bar{x}_{i}}}\left(\hat{\bar{x}}_{i}, t\right)\left[\dot{\bar{x}}_{i}+\alpha_{\bar{x}_{i}}(t) \bar{x}_{i}\right]
$$


where

$$
\begin{gathered}
\mathcal{J}_{T_{\bar{x}_{i}}\left(\hat{\bar{x}}_{i}, t\right) \triangleq} \frac{\partial T_{\bar{x}_{i}}\left(\hat{\bar{x}}_{i}\right)}{\partial \hat{\bar{x}}_{i}} \frac{1}{\rho_{\bar{x}_{i}}(t)}>0 \\
\alpha_{\bar{x}_{i}}(t) \triangleq-\frac{\dot{\rho}_{\bar{x}_{i}}(t)}{\rho_{\bar{x}_{i}}(t)}>0
\end{gathered}
$$

are the normalized Jacobian of the transformed function $T_{\bar{x}_{i}}$ and the normalized derivative of the performance function, respectively.

\section{Problem Statement}

We aim to design a control strategy for the leader-follower multi-agent system (4) such that it can achieve the target formation $\mathcal{F}$ as in (1) and the evolution of the relative positions between neighbouring agents should satisfy the prescribed performance bounds (5).

Problem 1. Let the leader-follower multi-agent system $\Sigma$ be defined by (4) with the communication graph $\mathcal{G}=(\mathcal{V}, \mathcal{E})$ and the prescribed performance functions $\rho_{\bar{x}_{i}}, i=1,2, \ldots, m$. Derive a control strategy such that the controlled leaderfollower multi-agent system achieves the target formation $\mathcal{F}$ as in (1) while satisfying (5).

\section{LEADER-FOLLOWER FORMATION CONTROL WITH PRESCRIBED PERFORMANCE GUARANTEES}

In this section, we design the control for the leaderfollower multi-agent system (4) such that the system can achieve the target formation $\mathcal{F}$ as in (1) within the prescribed performance bounds (5). The respective performance functions $\rho_{\bar{x}_{i}}(t)$ and transformed functions $T_{\bar{x}_{i}}\left(\hat{\bar{x}}_{i}\right)$ are defined as (7) and (8) without loss of generality. We assume that communicating agents share information about their performance functions $\rho_{\bar{x}_{i}}(t)$ and transformed functions $T_{\bar{x}_{i}}\left(\hat{\bar{x}}_{i}\right)$. Therefore, the communication between neighbouring agents is bidirectional and the graph $\mathcal{G}$ is assumed undirected.

Since our target is the relative position-based formation and, we first rewrite the dynamics (4) into the edge space to characterise the dynamics of the relative positions. We then rewrite (4) into the dynamics corresponding to followers and leaders, respectively. In addition, since we consider a general graph with circles that can be regarded as the union of two edge-disjoint subgraphs on the same vertex set as $\mathcal{G}=\mathcal{G}_{t} \cup \mathcal{G}_{c}$, where $\mathcal{G}_{t}$ is a spanning tree subgraph and $\mathcal{G}_{c}$ contains the remaining edges that necessarily complete the cycles in $\mathcal{G}$ [15]. The corresponding incidence matrix is decomposed as follows

$$
D=\left[\begin{array}{cc}
D_{F}^{t} & D_{F}^{c} \\
D_{L}^{t} & D_{L}^{c}
\end{array}\right], D^{t}=\left[\begin{array}{c}
D_{F}^{t} \\
D_{L}^{t}
\end{array}\right], D^{c}=\left[\begin{array}{c}
D_{F}^{c} \\
D_{L}^{c}
\end{array}\right],
$$

and $D_{F}=\left[\begin{array}{ll}D_{F}^{t} & D_{F}^{c}\end{array}\right], D_{L}=\left[\begin{array}{ll}D_{L}^{t} & D_{L}^{c}\end{array}\right]$ with $D_{F}^{t} \in$ $\mathbb{R}^{n_{f} \times(n-1)}, D_{F}^{c} \in \mathbb{R}^{n_{f} \times(m-n+1)}, D_{L}^{t} \in \mathbb{R}^{n_{l} \times(n-1)}, D_{L}^{c} \in$ $\mathbb{R}^{n_{l} \times(m-n+1)}$. That is, the incidence matrix $D$ is decomposed by the rows into the first $n_{f}$ and the remaining last $n_{l}$ rows, i.e., $D=\left[\begin{array}{ll}D_{F}^{T} & D_{L}^{T}\end{array}\right]^{T}$ [10] with $D_{F}, D_{L}$ denoting the incidence matrices with respect to the followers and leaders, respectively. Moreover, it is also decomposed by the columns into the first $n-1$ and the remaining last $m-n+1$ columns, i.e., $D=\left[\begin{array}{ll}D^{t} & D^{c}\end{array}\right]$ with $D^{t}, D^{c}$ denoting the edges of the spanning tree subgraph $\mathcal{G}_{t}$ and the remaining edges of $\mathcal{G}_{c}$ that necessarily complete the cycles in $\mathcal{G}$, respectively. Using $x=p-p^{d e s}$ and multiplying with $D^{T}$ on both sides of (4), then the dynamics (4) are reorganised into the edge space as

$$
\Sigma_{e}: \dot{\bar{x}}=-L_{e} \bar{x}+D_{L}^{T} u,
$$

with $L_{e}$ being the edge Laplacian which is positive definite if the graph is a tree [4]. Hence, we have that the edge Laplacian $L_{e}^{t}=D^{t^{T}} D^{t}$ for the spanning tree subgraph $\mathcal{G}_{t}$ is positive definite. For a general graph with cycles, the edge Laplacian $L_{e}$ in (13) is only positive semi-definite, but it can be represented by the edge Laplacian $L_{e}^{t}$ of its spanning tree $\mathcal{G}_{t}$ according to [15]. Specifically, the columns of $D^{c}$ are linearly dependent on the columns of $D^{t}$, which can be expressed as $D^{c}=D^{t} T$ with $T=$ $\left(D^{t^{T}} D^{t}\right)^{-1} D^{t^{T}} D^{c}=\left(L_{e}^{t}\right)^{-1} D^{t^{T}} D^{c}$. Then we have that $D=\left[\begin{array}{ll}D^{t} & D^{c}\end{array}\right]=\left[\begin{array}{ll}D^{t} & D^{t} T\end{array}\right]=D^{t}\left[\begin{array}{ll}I_{n-1} & T\end{array}\right]$. We further denote the transformation matrix $R$ as

$$
R=\left[\begin{array}{ll}
I_{n-1} & T
\end{array}\right] \text {. }
$$

Thus $D=D^{t} R$ and the relation between $L_{e}$ and $L_{e}^{t}$ is derived as $L_{e}=R^{T} L_{e}^{t} R$.

For the edge dynamics (13), the proposed controller applied to the leader agents is the composition of the term based on the prescribed performance of the positions of the neighbouring agents:

$$
u_{j}=-\sum_{i \in \Phi_{j}} g_{\bar{x}_{i}} \mathcal{J}_{T_{\bar{x}_{i}}}\left(\hat{\bar{x}}_{i}, t\right) \varepsilon_{\bar{x}_{i}}\left(\hat{\bar{x}}_{i}\right), \quad j \in \mathcal{V}_{L}
$$

where $\Phi_{j}=\left\{i \mid(j, k)=i, k \in \mathcal{N}_{j}\right\}$, i.e., the set of all the edges that include agent $j \in \mathcal{V}_{L}$ as a node, and $g_{\bar{x}_{i}}$ is a positive scalar gain to be appropriately tuned. Then the stack input vector is

$$
u=-D_{L} \mathcal{J}_{T_{\hat{x}}} G_{\bar{x}} \varepsilon_{\hat{x}}
$$

where $\hat{\bar{x}} \in \mathbb{R}^{m}$ is the stack vector of transformed errors $\hat{\bar{x}}_{i}, G_{\bar{x}} \in \mathbb{R}^{m \times m}$ is the positive definite diagonal gain matrix with entries the positive constant parameters $g_{\bar{x}_{i}}$, $\mathcal{J}_{T_{\hat{x}}} \triangleq \mathcal{J}_{T}(\hat{\bar{x}}, t) \in \mathbb{R}^{m \times m}$ is a time varying diagonal matrix with diagonal entries $\mathcal{J}_{T_{\bar{x}_{i}}}\left(\hat{\bar{x}}_{i}, t\right)$ given in (10), and $\varepsilon_{\hat{x}} \triangleq \varepsilon(\hat{\bar{x}}) \in \mathbb{R}^{m}$ is the stack vector with entries $\varepsilon_{\bar{x}_{i}}\left(\hat{\bar{x}}_{i}\right)$. Then the edge dynamics (13) with input (16) can be written as

$$
\dot{\bar{x}}=-L_{e} \bar{x}-D_{L}^{T} D_{L} \mathcal{J}_{T_{\hat{x}}} G_{\bar{x}} \varepsilon_{\hat{x}} .
$$

In the sequel, we develop the following result and will use Lyapunov-like methods to prove that the target formation can be achieved and the prescribed performance can be guaranteed.

Theorem 1. Consider the leader-follower multi-agent system $\Sigma$ with dynamics (4), the predefined performance functions $\rho_{\bar{x}_{i}}$ as in (7) and the transformed functions $T_{\bar{x}_{i}}\left(\hat{\bar{x}}_{i}\right)$ as in (8) s.t. $T_{\bar{x}_{i}}(0)=0$, and assume that the initial conditions $\bar{p}_{i}(0)$ are within the performance bounds (5). If the following condition holds:

$$
\bar{\gamma} \geq l=\max _{i=1, \ldots, m}\left(l_{\bar{x}_{i}}\right),
$$


where $l$ is the largest decay rate of $\rho_{\bar{x}_{i}}(t)$ and $\bar{\gamma}$ is the maximum value of $\gamma$ that ensures the following block matrix denoted as $\Gamma$ satisfies $\Gamma \geq 0$ :

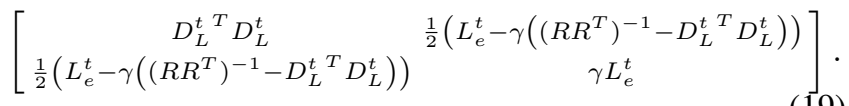

Then the controlled system achieves the target formation (1) and satisfies (5) when applying the control law (16).

Proof. Since the initial conditions $\bar{p}_{i}(0)$ are within the performance bounds (5), this is equivalent to that the initial conditions $\bar{x}_{i}(0)$ are within the performance bounds (6). Consider the Lyapunov-like function

$$
V\left(\varepsilon_{\hat{\bar{x}}}, \bar{x}\right)=\frac{1}{2} \varepsilon_{\overline{\hat{x}}}^{T} G_{\bar{x}} \varepsilon_{\hat{\bar{x}}}+\frac{\gamma}{2} \bar{x}^{T} \bar{x} .
$$

Then, $\dot{V}=\varepsilon_{\hat{\bar{x}}}^{T} G_{\bar{x}} \dot{\varepsilon}_{\hat{\bar{x}}}+\gamma \bar{x}^{T} \dot{\bar{x}}$. Replacing $\dot{\varepsilon}_{\hat{\bar{x}}}$ by stacking the components $\dot{\varepsilon}_{\bar{x}_{i}}\left(\hat{\bar{x}}_{i}\right)$ that are derived in (9), we obtain $\dot{V}=$ $\varepsilon_{\hat{\bar{x}}}^{T} G_{\bar{x}} \mathcal{J}_{T_{\hat{x}}}\left(\dot{\bar{x}}+\alpha_{\bar{x}}(t) \bar{x}\right)+\gamma \bar{x}^{T} \dot{\bar{x}}$, where $\alpha_{\bar{x}}(t)$ is the diagonal matrix with diagonal entries $\alpha_{\bar{x}_{i}}(t)$. According to (11) and (7), we know that $\alpha_{\bar{x}_{i}}(t)<l_{\bar{x}_{i}}, \forall t$. We then partition the edge state vector in (17) as $\bar{x}=\left[\begin{array}{ll}\bar{x}_{t}^{T} & \bar{x}_{c}^{T}\end{array}\right]^{T}$ with respect to the spanning tree and the cycles and get that

$$
\begin{aligned}
\bar{x} & =\left[\begin{array}{c}
\bar{x}_{t} \\
\bar{x}_{c}
\end{array}\right]=D^{T} x=\left[\begin{array}{c}
D^{t^{T}} \\
D^{c T}
\end{array}\right] x=\left[\begin{array}{c}
D^{t^{T}} \\
T^{T} D^{t^{T}}
\end{array}\right] x \\
& =\left[\begin{array}{c}
I_{n-1} \\
T^{T}
\end{array}\right] D^{t^{T}} x=\left[\begin{array}{c}
I_{n-1} \\
T^{T}
\end{array}\right] \bar{x}_{t}=R^{T} \bar{x}_{t} .
\end{aligned}
$$

We can further rewrite (17) as

$$
R^{T} \dot{\bar{x}}_{t}=-R^{T} L_{e}^{t} R R^{T} \bar{x}_{t}-R^{T} D_{L}^{t}{ }^{T} D_{L}^{t} R \mathcal{J}_{T_{\hat{\bar{x}}}} G_{\bar{x}} \varepsilon_{\hat{\bar{x}}} .
$$

Substituting (21) into $\dot{V}$, we can further derive that

$$
\begin{aligned}
\dot{V}= & \varepsilon_{\hat{\bar{x}}}^{T} G_{\bar{x}} \mathcal{J}_{T_{\hat{x}}}\left(-R^{T} L_{e}^{t} R R^{T} \bar{x}_{t}-R^{T} D_{L}^{t}{ }^{T} D_{L}^{t} R \mathcal{J}_{T_{\hat{x}}} G_{\bar{x}} \varepsilon_{\hat{\bar{x}}}\right. \\
& \left.+\alpha_{\bar{x}}(t) R^{T} \bar{x}_{t}\right)+\gamma \bar{x}_{t}^{T} R\left(-R^{T} L_{e}^{t} R R^{T} \bar{x}_{t}\right. \\
& \left.-R^{T} D_{L}^{t} D_{L}^{t} R \mathcal{J}_{T_{\hat{x}}} G_{\bar{x}} \varepsilon_{\hat{\bar{x}}}\right) \\
= & -\varepsilon_{\hat{\bar{x}}}^{T} G_{\bar{x}} \mathcal{J}_{T_{\hat{x}}} R^{T} L_{e}^{t} R R^{T} \bar{x}_{t}+\varepsilon_{\hat{\bar{x}}}^{T} G_{\bar{x}} \mathcal{J}_{T_{\hat{\bar{x}}}} \alpha_{\bar{x}}(t) \bar{x} \\
& -\varepsilon_{\hat{\bar{x}}}^{T} G_{\bar{x}} \mathcal{J}_{T_{\hat{x}}} R^{T} D_{L}^{t} D_{L}^{t} R \mathcal{J}_{T_{\hat{x}}} G_{\bar{x}} \varepsilon_{\hat{\bar{x}}} \\
& -\gamma \bar{x}_{t}^{T} R R^{T} L_{e}^{t} R R^{T} \bar{x}_{t} \\
& -\gamma \bar{x}_{t}^{T} R R^{T} D_{L}^{t} D_{L}^{t} R \mathcal{J}_{T_{\hat{x}}} G_{\bar{x}} \varepsilon_{\hat{\bar{x}}}
\end{aligned}
$$

Adding and subtracting $\gamma \varepsilon_{\hat{\bar{x}}}^{T} G_{\bar{x}} \mathcal{J}_{T_{\hat{x}}} R^{T} \bar{x}_{t}$ on the right hand side of (22), we obtain

$$
\begin{aligned}
\dot{V}= & -\varepsilon_{\hat{\bar{x}}}^{T} G_{\bar{x}} \mathcal{J}_{T_{\hat{x}}}\left(\gamma I_{m}-\alpha_{\bar{x}}(t)\right) \bar{x}-\varepsilon_{\hat{\bar{x}}}^{T} G_{\bar{x}} \mathcal{J}_{T_{\hat{x}}} R^{T} L_{e}^{t} R R^{T} \bar{x}_{t} \\
& -\varepsilon_{\hat{\bar{x}}}^{T} G_{\bar{x}} \mathcal{J}_{T_{\hat{x}}} R^{T} D_{L}^{t} D_{L}^{t} R \mathcal{J}_{T_{\hat{x}}} G_{\bar{x}} \varepsilon_{\hat{\bar{x}}} \\
& -\gamma \bar{x}_{t}^{T} R R^{T} L_{e}^{t} R R^{T} \bar{x}_{t} \\
& +\gamma \bar{x}_{t}^{T} R R^{T}\left(\left(R R^{T}\right)^{-1}-D_{L}^{t}{ }^{T} D_{L}^{t}\right) R \mathcal{J}_{T_{\hat{x}}} G_{\bar{x}} \varepsilon_{\hat{\bar{x}}} \\
= & -\varepsilon_{\hat{\bar{x}}}^{T} G_{\bar{x}} \mathcal{J}_{T_{\hat{x}}}\left(\gamma I_{m}-\alpha_{\bar{x}}(t)\right) \bar{x}-y^{T} \Gamma y
\end{aligned}
$$

with $y=\left[\begin{array}{ll}\varepsilon_{\hat{x}}^{T} G_{\bar{x}} \mathcal{J}_{T_{\hat{x}}} R^{T} & \bar{x}^{T} R R^{T}\end{array}\right]^{T}$ and $\Gamma$ described as (19). We have that $G_{\bar{x}} \mathcal{J}_{T_{\hat{x}}}$ is a diagonal positive definite matrix; $\left(\gamma I_{m}-\alpha_{\bar{x}}(t)\right)$ is a diagonal positive definite matrix if $\gamma \geq l=\max \left(l_{\bar{x}_{i}}\right)>\bar{\alpha}=\sup \alpha_{\bar{x}_{i}}(t)$. Since the transformed function $T_{\bar{x}_{i}}\left(\hat{\bar{x}}_{i}\right)$ is strictly increasing and $T_{\bar{x}_{i}}(0)=0$, we have $\varepsilon_{\bar{x}_{i}}\left(\hat{\bar{x}}_{i}\right) \hat{\bar{x}}_{i}=T_{\bar{x}_{i}}\left(\hat{\bar{x}}_{i}\right) \hat{\bar{x}}_{i} \geq 0$. Therefore, we get that $-\varepsilon_{\hat{\bar{x}}}^{T} G_{\bar{x}} \mathcal{J}_{T_{\hat{x}}}\left(\gamma I_{m}-\alpha_{\bar{x}}(t)\right) \bar{x} \leq 0$ holds. Then, in order for $\dot{V} \leq 0$ to hold, it suffices that $\gamma \geq l=\max \left(l_{\bar{x}_{i}}\right)>$ $\sup \alpha_{\bar{x}_{i}}(t)$ and in addition, $\Gamma$ should be positive semidefinite. Then, based on condition (18), and choosing $\gamma=\bar{\gamma}$, we obtain $-\varepsilon_{\hat{\bar{x}}}^{T} G_{\bar{x}} \mathcal{J}_{T_{\hat{\bar{x}}}}\left(\bar{\gamma} I_{m}-\alpha_{\bar{x}}(t)\right) \bar{x} \leq 0$ and $\Gamma \geq 0$. Finally, we can conclude that $\dot{V} \leq 0$ when $\gamma=\bar{\gamma}$. This also implies $V\left(\varepsilon_{\hat{\bar{x}}}, \bar{x}\right) \leq V\left(\varepsilon_{\hat{\bar{x}}}(0), \bar{x}(0)\right)$. Hence if $\left.\bar{x}(0)\right)$ is chosen within the region $\mathcal{D}_{\bar{x}_{i}}$ then $V\left(\varepsilon_{\hat{\bar{x}}}(0), \bar{x}(0)\right)$ is finite, which implies that $V\left(\varepsilon_{\hat{\hat{x}}}, \bar{x}\right)$ is bounded $\forall t$. Therefore $\varepsilon_{\hat{\hat{x}}}, \bar{x}$ are bounded and the boundedness of $\varepsilon_{\hat{\bar{x}}}$ implies that the relative position $\bar{x}(t)$ evolves within the prescribed performance bounds (6), $\forall t$. Then we can deduce the boundedness of $\ddot{V}\left(\varepsilon_{\hat{\bar{x}}}, \bar{x}\right)$ based on the boundedness of $\varepsilon_{\hat{\bar{x}}}, \dot{\varepsilon}_{\hat{\bar{x}}}$. The boundedness of $\ddot{V}\left(\varepsilon_{\hat{\bar{x}}}, \bar{x}\right)$ implies the uniform continuity of $\dot{V}\left(\varepsilon_{\hat{\bar{x}}}, \bar{x}\right)$, which in turn implies that $\dot{V}\left(\varepsilon_{\hat{x}}, \bar{x}\right) \rightarrow 0$ as $t \rightarrow \infty$ by applying Barbalat's Lemma. This implies $\bar{x} \rightarrow 0$ as $t \rightarrow \infty$, which also means that $\bar{p} \rightarrow \bar{p}^{\text {des }}$ as $t \rightarrow \infty$. Hence, the target formation (1) is achieved while satisfying (5).

Remark 1. Note that conditions (18) and (19) are not part of the control laws. (19) is determined by the matrices $L_{e}^{t}, D_{L}^{t}, R$ that characterise the leader-follower graph topology, thus the existence of $\gamma$ is determined by the leader-follower graph topology. According to Theorem 1, we can first solve (19) to obtain the maximum value $\bar{\gamma}$ of $\gamma$ that ensures $\Gamma \geq 0$. Then, the predefined largest decay rate $l$ of performance functions $\rho_{\bar{x}_{i}}(t)$ cannot exceed this value $\bar{\gamma}$. Nevertheless, Theorem 1 can be useful in practical applications to predesign the maximum exponential decay rate of the performance functions.

Remark 2. The result in this section indicates the trade-offs between the largest decay rate of the performance functions (7) and the leader amount and positions. Theorem 1 provides a sufficient condition for a general graph with cycles, under which the leader-follower multi-agent system (4) achieves the target relative position-based formation within the prescribed performance bounds (5). It can be seen that (18) may be infeasible when the decay rate of the performance functions (7) is too large. This means that we need to constrain the decay rate of the performance functions in order to achieve the target formation under prescribed performance guarantees within the leader-follower framework. This is reasonable since the followers only obey the first-order formation protocol without any additional external input.

Remark 3. Theorem 1 also indicates how the cycles in a graph will benefit the convergence result. Compared with our previous work [2], we consider general graphs with cycles here, which extends earlier results on trees. When $R=I_{n-1}$, in this case the communication graph is a tree and Theorem 1 is exactly the result for tree graphs in [2]. Here, Theorem 1 indicates that once the spanning tree $\mathcal{G}_{t}$ can achieve the target formation within the prescribed performance bounds, then the whole graph $\mathcal{G}$ that contains the cycles and with the 
same leader set $\mathcal{V}_{L}$ can also achieve the target formation without violating the prescribed performance bounds. This means that adding cycles to the spanning tree will preserve convergence and even benefit the convergence in the sense that the prescribed performance bounds can have a higher decay rate.

\section{NECESSARY CONDITIONS ON THE GRAPH TOPOLOGY}

In this section, we derive necessary conditions on the graph topologies for both tree graphs and general graphs with cycles such that under these conditions we can design the leaders to achieve the target formation with prescribed performance guarantees.

We first discuss the tree graphs and then the results for general graphs with cycles are built based on the results of tree graphs. We first define a graph $\mathcal{G}^{f}=\left(\mathcal{V}^{f}, \mathcal{E}^{f}\right)$ with only followers, i.e., $\mathcal{V}_{F}^{f}=\mathcal{V}^{f}, \mathcal{V}_{L}^{f}=\emptyset$ and $\mathcal{E}_{F F}^{f}=\mathcal{E}^{f}$. Suppose that the leader-follower multi-agent system described by the graph $\mathcal{G}=(\mathcal{V}, \mathcal{E})$ contains $\mathcal{G}^{f}$ as a subgraph. Here, a subgraph is defined as follows:

Definition 1. A graph $\mathcal{G}^{\prime}=\left(\mathcal{V}^{\prime}, \mathcal{E}^{\prime}\right)$ is a subgraph of the graph $\mathcal{G}=(\mathcal{V}, \mathcal{E})$ if the following conditions hold:

- $\mathcal{V}^{\prime} \subseteq \mathcal{V}$ and $\mathcal{E}^{\prime} \subseteq \mathcal{E}$;

- for any $i \in \mathcal{V}_{F}^{\prime}$ we have $i \in \mathcal{V}_{F}$ and for any $i \in \mathcal{V}_{L}^{\prime}$ we have $i \in \mathcal{V}_{L}$;

- for any edge $(i . j) \in \mathcal{E}, i, j \in \mathcal{V}^{\prime}$, we have $(i . j) \in \mathcal{E}^{\prime}$.

From now on, we denote $\bar{x}$ as the edge state of $\mathcal{G}^{f}$ and $L_{e}$ as the edge Laplacian of $\mathcal{G}^{f}$. We know that the edge dynamics of $\mathcal{G}^{f}$ are simply described as $\dot{\bar{x}}=-L_{e} \bar{x}$ since the leader set $\mathcal{V}_{L}^{f}$ is empty. Denote each column (corresponding to an edge) of the incidence matrix of $\mathcal{G}^{f}$ by the vector $e_{i}$. Then $\left(L_{e}\right)_{i j}=e_{i}^{T} e_{j}=c_{i j}=2$ if $i=j ; c_{i j}=0$ if $e_{i}, e_{j}$ share no nodes; $c_{i j}=1$ if $e_{i}, e_{j}$ share a single node and have the same direction with respect to the sharing node (i.e., both inward or outward the node); $c_{i j}=-1$ if $e_{i}, e_{j}$ share a single node but have different direction with respect to the sharing node [15]. Based on this, we define the neighbor of edge $e_{i}$ as $\mathcal{N}\left(e_{i}\right):=\left\{e_{j}|| e_{i}^{T} e_{j} \mid=1\right\}$. Then the following theorem is developed for tree graphs, which proposes a necessary condition on the graph, under which we can design the leaders to achieve the target formation with prescribed performance guarantees.

Theorem 2. Consider the leader-follower multi-agent system $\Sigma$ described by the tree graph $\mathcal{G}=(\mathcal{V}, \mathcal{E})$, a necessary condition on $\mathcal{G}$ under which we can design the leaders using (16) to achieve the target formation $\mathcal{F}$ as in (1) while satisfying (5) is that: $\mathcal{G}$ does not contain a subgraph $\mathcal{G}^{f}=\left(\mathcal{V}^{f}, \mathcal{E}^{f}\right)$ with $\mathcal{V}_{F}^{f}=\mathcal{V}^{f}$ such that there exists $e_{i} \in \mathcal{E}^{f}$ satisfying $\left|\mathcal{N}\left(e_{i}\right)\right| \geq 3$.

Proof. The proof uses contradiction based on the entries of the edge Laplacian $L_{e}$ of $\mathcal{G}^{f}$. Suppose $\mathcal{G}^{f}$ is a subgraph of $\mathcal{G}$ and there exists $e_{i} \in \mathcal{E}^{f}$ satisfying $\left|\mathcal{N}\left(e_{i}\right)\right| \geq 3$. Without loss of generality, let us assume that $e_{1} \in \mathcal{E}^{f}$ satisfies $\mathcal{N}\left(e_{1}\right)=$ $\left\{e_{2}, e_{3}, e_{4}\right\}$ thus $\left|\mathcal{N}\left(e_{i}\right)\right|=3$. Suppose that $e_{2}, e_{3}, e_{4}$ all share a single node with $e_{1}$ but with different directions.
This can be assumed without loss of generality since we can assign arbitrary directions to the edges. Then the state evolution of $e_{1}$ is derived as $\dot{\bar{x}}_{1}=-2 \bar{x}_{1}+\bar{x}_{2}+\bar{x}_{3}+\bar{x}_{4}$. We can see that when all $\bar{x}_{i}, i=1,2,3,4$ are initialised arbitrarily close to the prescribed performance boundary $\rho_{\bar{x}_{i 0}}=\rho_{0}, i=1,2,3,4$, then $\dot{\bar{x}}_{1}=\rho_{0}>0$, thus $\bar{x}_{1}$ will continue evolving to violate the performance bound. This leads to a contradiction since no matter how we design the leaders in $\mathcal{G} \backslash \mathcal{G}_{t}, \bar{x}_{1}$ will always increase to violate the boundary. Hence, we can conclude that $\mathcal{G}$ should not contain a subgraph $\mathcal{G}^{f}=\left(\mathcal{V}^{f}, \mathcal{E}^{f}\right)$ with $\mathcal{V}_{F}^{f}=\mathcal{V}^{f}$ such that there exists $e_{i} \in \mathcal{E}^{f}$ satisfying $\left|\mathcal{N}\left(e_{i}\right)\right| \geq 3$.

Next, we generalise Theorem 2 to derive a necessary condition for graphs with cycles under which we can design the leaders to achieve the target formation without violating the prescribed performance bounds. First of all, we perform the following graph decomposition and call as complete decomposition.

Definition 2. A graph $\mathcal{G}=(\mathcal{V}, \mathcal{E})$ is decomposed as $\mathcal{G}=$ $\cup_{i=1}^{k} \mathcal{C}_{i} \cup \mathcal{P}$, where $\mathcal{C}_{i}, i=1, \ldots, k$ are cycles and each $\mathcal{C}_{i}$ does not contain a cycle subgraph that has less edges than $\mathcal{C}_{i} ; \mathcal{P}$ is the set of the edges that do not belong to any cycle. Then, we call each $\mathcal{C}_{i}$ a minimal cycle and this decomposition a complete decomposition of $\mathcal{G}$.

The complete decomposition decomposes a large scale graph into some minimal cycles together with the remaining edges that do not belong to any cycle. We can then derive the necessary condition for $\mathcal{G}$ based on the decomposed minimal cycles $\mathcal{C}_{i}$ and the remaining edges $\mathcal{P}$. We denote here $\mathcal{E}\left(\mathcal{C}_{i}\right)$ as the edge set of the minimal cycle $\mathcal{C}_{i}$ with cardinality $\left|\mathcal{E}\left(\mathcal{C}_{i}\right)\right|$. We further define a function $f(x), x \geq 3, x \in \mathbb{Z}$, which will be used later to derive the necessary conditions for general graphs with cycles:

$$
f(x)=\left\{\begin{array}{lll}
x, & 3 \leq x \leq 6, & x \in \mathbb{Z} ; \\
6, & x \geq 7, & x \in \mathbb{Z} .
\end{array}\right.
$$

Now we derive the following theorem that proposes a necessary condition on general graphs with cycles, under which we can design the leaders to achieve the target formation without violating the prescribed performance bounds.

Theorem 3. Consider the leader-follower multi-agent system $\Sigma$ described by the graph $\mathcal{G}=(\mathcal{V}, \mathcal{E})$, a necessary condition on $\mathcal{G}$ under which we can design the leaders using (16) to achieve the target formation $\mathcal{F}$ as in (1) while satisfying (5) is that: $\mathcal{G}$ does not contain a subgraph $\mathcal{G}^{f}=\left(\mathcal{V}^{f}, \mathcal{E}^{f}\right)$ with $\mathcal{V}_{F}^{f}=\mathcal{V}^{f}$ and completely decomposed as $\mathcal{G}^{f}=\cup_{i=1}^{k} \mathcal{C}_{i} \cup \mathcal{P}$ such that there exists $e_{i} \in \mathcal{E}^{f}$ satisfying

$$
\sum_{j \in S_{i}}\left(f\left(\left|\mathcal{E}\left(\mathcal{C}_{j}\right)\right|\right)-4\right)+\left|E_{i}\right| \geq 3,
$$

where $S_{i}=\left\{j \mid e_{i} \in \mathcal{E}\left(\mathcal{C}_{j}\right)\right\}, E_{i}=\left\{e_{k} \mid e_{k} \in \mathcal{N}\left(e_{i}\right), e_{k} \notin\right.$ $\left.\mathcal{C}_{j}, j \in S_{i}\right\}$ and the function $f(x)$ is defined as (24).

Proof. The proof is based on the discussion of the decomposed minimal cycles $\mathcal{C}_{i}$ and the remaining edges $\mathcal{P}$. We 
can resort to Theorem 2 to deal with $\mathcal{P}$. Here, we first discuss the result for a minimal cycle, e.g., $\mathcal{C}_{1}$ which has $m$ edges $\mathcal{E}\left(\mathcal{C}_{1}\right)=\left\{e_{1}, e_{2}, \ldots, e_{m}\right\}$ and $m \geq 3$. We will check how the number of edges of the cycle affects convergence. When $m=3$, the state evolution of an arbitrary edge $e_{1}$ is $\dot{\bar{x}}_{1}=-2 \bar{x}_{1}+\bar{x}_{2}+\bar{x}_{3}$, and since $e_{i}, i=1,2,3$ form a cycle, we have that $\bar{x}_{1}+\bar{x}_{2}+\bar{x}_{3}=0$. Hence $\bar{x}_{2}+\bar{x}_{3}=-\bar{x}_{1}$ and $\dot{\bar{x}}_{1}=-2 \bar{x}_{1}-\bar{x}_{1}=-3 \bar{x}_{1}$. This means that the cycle that forms a triangle will show a higher convergence rate of -3 for the edge dynamics. When $m=4$, the state evolution of an arbitrary edge $e_{1}$ is $\dot{\bar{x}}_{1}=-2 \bar{x}_{1}+\bar{x}_{2}+\bar{x}_{4}$, since $e_{i}, i=1,2,3,4$ form a cycle, we have that $\sum_{i=1}^{4} \bar{x}_{i}=0$. Hence $\bar{x}_{2}+\bar{x}_{4}=-\bar{x}_{1}-\bar{x}_{3}$ and $\dot{\bar{x}}_{1}=-2 \bar{x}_{1}-\bar{x}_{1}-\bar{x}_{3}$. Consider the limit case, when $\bar{x}_{1}$ is arbitrarily close to the prescribed performance boundary and $\bar{x}_{3}=-\bar{x}_{1}$, we have $\dot{\bar{x}}_{1}=-2 \bar{x}_{1}-\bar{x}_{1}+\bar{x}_{1}=-2 \bar{x}_{1}$. This means that the cycle which forms a square still show decay rate of -2 for the edge dynamics. When $m=5$, the state evolution of an arbitrary edge $e_{1}$ is $\dot{\bar{x}}_{1}=-2 \bar{x}_{1}+\bar{x}_{2}+\bar{x}_{5}$, since $e_{i}, i=1,2,3,4,5$ form a cycle, we have that $\sum_{i=1}^{5} \bar{x}_{i}=0$. Hence $\bar{x}_{2}+\bar{x}_{5}=-\bar{x}_{1}-\bar{x}_{3}-\bar{x}_{4}$ and $\dot{\bar{x}}_{1}=-2 \bar{x}_{1}-\bar{x}_{1}-\bar{x}_{3}-\bar{x}_{4}$. Consider the limit case, when $\bar{x}_{1}$ is arbitrarily close to the performance bound and $\bar{x}_{3}=\bar{x}_{4}=-\bar{x}_{1}$, we then have $\dot{\bar{x}}_{1}=-2 \bar{x}_{1}-\bar{x}_{1}+2 \bar{x}_{1}=-\bar{x}_{1}$. This means that the cycle with 5 edges still show decay rate of -1 for the edge dynamics. This means that we still have the freedom to add one more edge that shares a node with $e_{1}$. For $m \geq 6$, the state evolution of an arbitrary edge $e_{1}$ is $\dot{\bar{x}}_{1}=-2 \bar{x}_{1}+\bar{x}_{2}+\bar{x}_{m}$, and since $e_{i}, i=1,2, \ldots, m$ forms a cycle, we have that $\sum_{i=1}^{m} \bar{x}_{i}=0$. Then $\bar{x}_{2}+\bar{x}_{m}=-\bar{x}_{1}-\sum_{i=3}^{m-1} \bar{x}_{i}$ and $\dot{\bar{x}}_{1}=$ $-2 \bar{x}_{1}-\bar{x}_{1}-\sum_{i=3}^{m-1} \bar{x}_{i} .-\sum_{i=3}^{m-1} \bar{x}_{i}$ cannot be greater than $3 \bar{x}_{1}$ when $\bar{x}_{1}$ is arbitrarily close to the performance bound since $e_{i}, i=1,2, \ldots, m$ need to form a cycle satisfying $\sum_{i=1}^{m} \bar{x}_{i}=0$. This means that for the cycle with $m \geq 6$, in the worst case we have $\dot{\bar{x}}_{1}=0$, thus in the limit case $\bar{x}_{1}$ will never evolve again to violate the performance boundary. Till here, we can summarise that for a single cycle $\mathcal{C}_{1}$, in the worst case the decay rate of the edge dynamics is $-2+f\left(\left|\mathcal{E}\left(\mathcal{C}_{1}\right)\right|\right)-4$ with the function $f(x)$ defined in $(24)$. Then, based on the result for a single minimal cycle, we build the result in the case that $e_{i}$ belongs to more than one minimal cycles, i.e., $e_{i}$ is an edge of $\mathcal{C}_{j}, j \in S_{i}$. Then each cycle $\mathcal{C}_{j}, j \in S_{i}$ will contribute a decay rate of $f\left(\left|\mathcal{E}\left(\mathcal{C}_{j}\right)\right|\right)-4$ for $e_{i}$. Since the cycles in $\mathcal{C}_{j}, j \in S_{i}$ will not affect each other in contributing to the decay rate of $e_{i}$ since they are minimal cycles and are completely decoupled with respect to $e_{i}$, then the total decay rate of $e_{i}$ that belongs to more than one minimal cycles is $-2+\sum_{j \in S_{i}}\left(f\left(\left|\mathcal{E}\left(\mathcal{C}_{j}\right)\right|\right)-4\right)$, where -2 corresponds to the diagonal entry of the edge Laplacian $-L_{e}$. Finally, the remaining edges that affect the convergence of $e_{i}$ are the edges that share a node with $e_{i}$ but not an edge of any $\mathcal{C}_{j}, j \in S_{i}$, i.e., $E_{i}=\left\{e_{k} \mid e_{k} \in \mathcal{N}\left(e_{i}\right), e_{k} \notin \mathcal{C}_{j}, j \in S_{i}\right\}$. Hence, $E_{i}$ is composed of the edges in $\mathcal{P}$ that share a node with $e_{i}$ and the edges that share a node with $e_{i}$ but belong to other minimal cycles that do not include $e_{i}$ as an edge. In the limit case, each of these edges will contribute a decay rate of 1 to edge $e_{i}$. Hence, the total decay rate of $e_{i}$ in the worst case is $-2+\sum_{j \in S_{i}}\left(f\left(\left|\mathcal{E}\left(\mathcal{C}_{j}\right)\right|\right)-4\right)+\left|E_{i}\right|$ and the violation condition is $-2+\sum_{j \in S_{i}}\left(f\left(\left|\mathcal{E}\left(\mathcal{C}_{j}\right)\right|\right)-4\right)+\left|E_{i}\right| \geq 1$, i.e., exactly the inequality (25). This means that in the worst case $e_{i}$ will continue evolving to violate the prescribed performance bounds. Therefore, suppose that there exists $e_{i} \in \mathcal{E}^{f}$ satisfying (25), then we can conclude that $e_{i}$ will evolve and violate the performance bound, which leads to a contradiction since no matter how we design the leaders in $\mathcal{G} \backslash \mathcal{G}_{t}, \bar{x}_{i}$ will always evolve to violate the prescribed performance bound. Hence, we can conclude that $\mathcal{G}$ should not contain a subgraph $\mathcal{G}^{f}=\left(\mathcal{V}^{f}, \mathcal{E}^{f}\right)$ with $\mathcal{V}_{F}^{f}=\mathcal{V}^{f}$ such that there exists $e_{i} \in \mathcal{E}^{f}$ satisfying (25).

Remark 4. Actually, Theorem 2 and Theorem 3 indicate that when considering the leader-follower multi-agent system $\Sigma$ to achieve the target formation within the prescribed performance bounds, we should avoid cases such that (25) holds when assigning leaders. They also propose a criterion in choosing leaders to achieve the target formation with prescribed performance guarantees, which can be further applied to solve leader selection problems. Note that Theorem 2 is a specific case of Theorem 3. That is, when the graph is a tree, then the term $\sum_{j \in S_{i}}\left(f\left(\left|\mathcal{E}\left(\mathcal{C}_{j}\right)\right|\right)-4\right)$ vanishes and $\left|E_{i}\right|$ is the number of edges that share a node with $e_{i}$, i.e., $\left|E_{i}\right|=\left|\mathcal{N}\left(e_{i}\right)\right|$. This also leads to the violation condition $\left|\mathcal{N}\left(e_{i}\right)\right| \geq 3$ for tree graphs.

\section{Simulations And EXAmples}

In this section, several simulation examples are presented in order to verify the results. The simulations' communication graphs are shown as Fig. 1A, where the leaders and followers are represented by grey and white nodes, respectively. We choose, without loss of generality, the same $\rho_{\bar{x}_{i}}$ for all edges as $\rho_{\bar{x}_{i}}(t)=4.9 e^{-t}+0.1$ and the same transformed function $T_{\bar{x}_{i}}$ as (8). In addition, the prescribed performance bounds are depicted in black color in the following simulation plots.
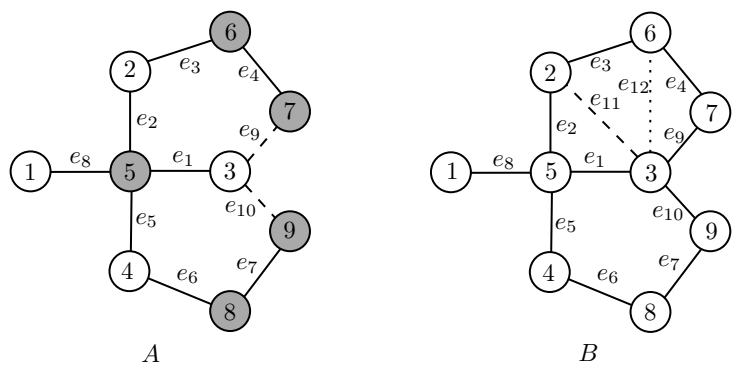

Fig. 1: Communication graphs.

In Fig. 1A, we first consider a tree graph with respective follower and leader set as $\mathcal{V}_{F}=\{1,2,3,4\}$ and $\mathcal{V}_{L}=\{5,6,7,8,9\}$; the edge set is $\mathcal{E}=\left\{e_{1}, \ldots, e_{8}\right\}$. The positions of all the agents are initialised at the origin and the target relative position-based formation is $p_{53}^{\text {des }}=$ $[-4.8,0]^{T}, p_{52}^{\text {des }}=[2.4,-4.8]^{T}, p_{62}^{\text {des }}=[4.8,4.8]^{T}, p_{76}^{\text {des }}=$ 
$[4.8,-4.8]^{T}, p_{54}^{\text {des }}=[2.4,4.8]^{T}, p_{84}^{\text {des }}=[4.8,-4.8]^{T}, p_{98}^{\text {des }}=$ $[4.8,4.8]^{T}, p_{51}^{\text {des }}=[4.8,0]^{T}$. The simulation result in two dimensions when applying the PPC law (16) is shown in Fig. 2. Here, all agents are initialised at origin and the blue lines show the final relative positions of the agents. The dashed lines indicate the evolution of the agents. It can be seen that the target formation is achieved. In order to verify whether the prescribed performance is guaranteed, we only plot the evolution of the relative positions for edges $e_{4}, e_{5}, e_{6}, e_{7}$ in ydirection due to space limitations, which are depicted in Fig. 3a. The red lines show the result without PPC, and we can see that the trajectories violate the prescribed performance bounds, which are improved by applying the PPC law (16). The result when applying PPC law is shown as the blue lines and we can observe that all the trajectories evolve within the prescribed performance bounds.

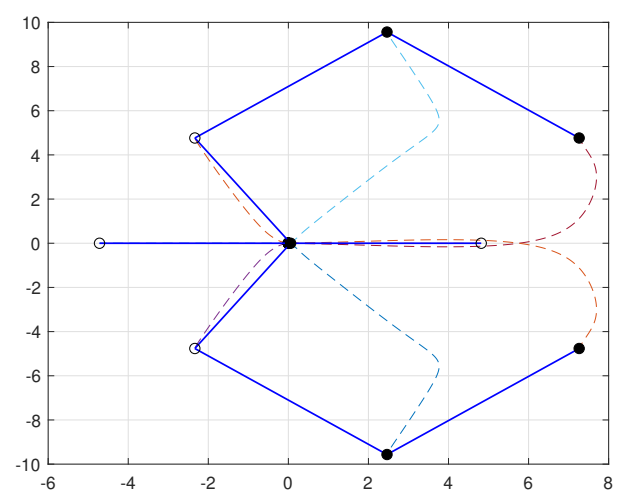

Fig. 2: Relative position-based formation control using PPC for the tree graph.

As a comparison, we add two edges into the tree graph, i.e., $e_{9}$ and $e_{10}$ to form two cycles in the communication graph as shown in Fig. 1A by the dashed lines. All agents
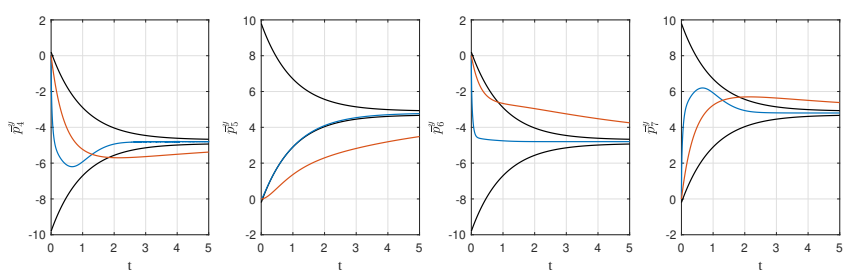

(a) Trajectories of the relative positions for the tree.
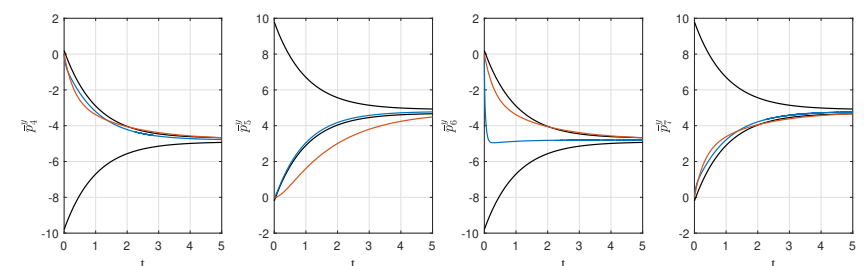

(b) Trajectories of the relative positions for the graph with cycles.

Fig. 3: Trajectories of relative positions for $e_{4}, e_{5}, e_{6}, e_{7}$ in y-direction. are still initialised at the origin and the target relative positions for edges $e_{1}, e_{2}, \ldots, e_{8}$ remain unchanged. We add the target relative positions for edges $e_{9}, e_{10}$ as $p_{73}^{\text {des }}=$ $[2.4,4.8]^{T}, p_{93}^{\text {des }}=[2.4,-4.8]^{T}$, which can also be derived based on target relative positions of $e_{1}, e_{2}, \ldots, e_{7}$ since they form the two cycles. The simulation result in two dimensions for the graph with cycles when applying the PPC law (16) is shown in Fig. 4. The target formation is also achieved. We note that the trajectories violate the prescribed performance bounds without PPC (red lines), which are improved by applying the PPC law (16) as shown by the blue lines. For both of the graphs, we can conclude that the controlled leader-follower multi-agent system achieves the target formation within the prescribed performance bound. We can also find that the graph with cycles converges to the target formation faster than the tree graph since the result of the tree graph shows a larger overshoot. Here, both of the decay rate of prescribed performance functions for the tree and the graph with cycles is 1 . This means that we do not observe a larger decay rate of the performance functions for the graph with cycles. This is due to that agent 5 connects with 4 followers, which restricts the maximum allowable decay rate of the performance functions. However, in order to achieve a higher decay rate, we can suitably assign more leaders or more cycles.

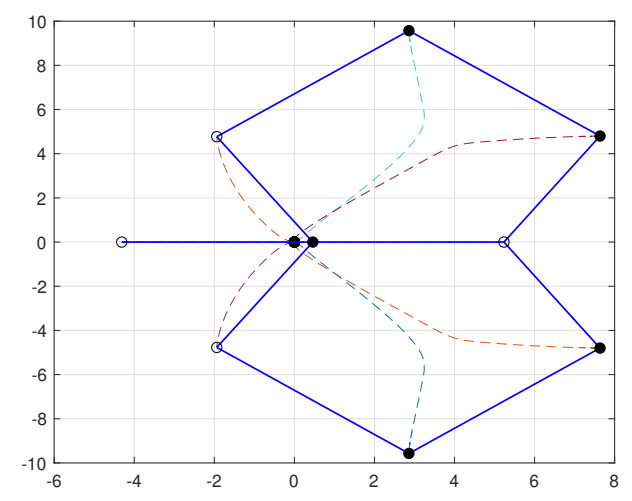

Fig. 4: Relative position-based formation control using PPC for the graph with cycles.

Finally, we consider an example to verify Theorem 2 and Theorem 3. We investigate the same graph that contains 2 minimal cycles as shown in Fig. 1B with all agents as followers, i.e., $\mathcal{G}^{f}=\left(\mathcal{V}^{f}, \mathcal{E}^{f}\right)$. We check how the leaderfollower multi-agent system described by the graph $\mathcal{G}=$ $(\mathcal{V}, \mathcal{E})$ will behave if it contains $\mathcal{G}^{f}$ as a subgraph. For $e_{8}$, it does not belong to any minimal cycle. Since $e_{8}$ is an edge of the star graph $\mathcal{G}^{s}=\left(\mathcal{V}^{s}, \mathcal{E}^{s}\right)$ with $\mathcal{V}^{s}=$ $\{1,2,3,4,5\}, \mathcal{E}^{s}=\left\{e_{1}, e_{2}, e_{5}, e_{8}\right\}$, which is a subgraph of $\mathcal{G}^{f}$. Based on Theorem 2, we have the violation condition $\left|\mathcal{N}\left(e_{1}\right)\right| \geq 3$ holds since $e_{8}$ has 3 neighboring edges. Hence, in the worst case, no matter how we design the leaders in $\mathcal{G}, e_{8}$ will violate the prescribed performance bounds (7). For $e_{2}$, it belongs to the minimal cycle $\mathcal{C}_{1}$ on the top and 
we have that $\left|\mathcal{E}\left(\mathcal{C}_{1}\right)\right|=5$. The number $\left|E_{2}\right|$ in (25) is 2 when considering the edge $e_{2}$, which is composed of edges $e_{5}, e_{8}$ because $e_{8}$ does not belong to any minimal cycle and $e_{5}$ belongs to the bottom minimal cycle $\mathcal{C}_{2}$ that does not contain $e_{2}$. Hence the violation condition holds for $e_{2}$ since $\sum_{j \in S_{2}}\left(f\left(\left|\mathcal{E}\left(\mathcal{C}_{j}\right)\right|\right)-4\right)+\left|E_{2}\right|=f\left(\left|\mathcal{E}\left(\mathcal{C}_{1}\right)\right|\right)-4+2=5-4+2 \geq$ 3. According to Theorem 3, we can conclude that in the worst case, no matter how we design the leaders in $\mathcal{G}, e_{2}$ will violate the prescribed performance bounds. We can derive a similar result for edge $e_{5}$ due to the graph symmetry. Then, we consider $e_{1}$, which belongs to both minimal cycles $\mathcal{C}_{1}, \mathcal{C}_{2}$. We derive that $\sum_{j \in S_{1}}\left(f\left(\left|\mathcal{E}\left(\mathcal{C}_{j}\right)\right|\right)-4\right)+\left|E_{1}\right|=f\left(\left|\mathcal{E}\left(\mathcal{C}_{1}\right)\right|\right)-$ $4+f\left(\left|\mathcal{E}\left(\mathcal{C}_{2}\right)\right|\right)-4+1=5-4+5-4+1 \geq 3$, thus the violation condition (25) holds. For the remaining edges, the violation condition does not hold according to Theorem 3. Next, if we only add an edge between agent 3 and 6 , i.e., $e_{12}$ (dotted line in Fig. 1B). Then $e_{2}$ voids the violation condition since $e_{2}$ belongs to a new minimal cycle with 4 edges, but the violation condition still holds for $e_{1}$ since $\sum_{j \in S_{1}}\left(f\left(\left|\mathcal{E}\left(\mathcal{C}_{j}\right)\right|\right)-4\right)+\left|E_{1}\right|=4-4+5-4+2 \geq 3$. Then, if we only add an edge between agent 2 and 3 , i.e., $e_{11}$ (dashed line in Fig. 1B). Then $e_{1}$ also voids the violation condition since now $\sum_{j \in S_{1}}\left(f\left(\left|\mathcal{E}\left(\mathcal{C}_{j}\right)\right|\right)-4\right)+\left|E_{1}\right|=3-4+5-4+2=2<3$.

One simple way for $\mathcal{G}^{f}$ to void the violation condition (25) is to cut the edge $e_{8}$.

\section{Conclusions}

In this paper, we have addressed relative position-based formation control problems of leader-follower multi-agent systems with prescribed performance bounds. The general case of graphs with cycles is treated and a distributed prescribed performance control law is proposed for the group of leaders in order to steer the followers such that the entire system can achieve the target formation under prescribed performance guarantees. For both tree graphs and general graphs with cycles, necessary conditions on the graph topologies are proposed such that under these conditions we can design the leaders to achieve the target formation with prescribed performance guarantees.

Future research includes applying other transient approaches and also investigating leader selection problems.

\section{REFERENCES}

[1] C. P. Bechlioulis and G. A. Rovithakis. Robust adaptive control of feedback linearizable mimo nonlinear systems with prescribed performance. IEEE Transactions on Automatic Control, 53(9):20902099, 2008.

[2] F. Chen and D. V. Dimarogonas. Consensus control for leader-follower multi-agent systems under prescribed performance guarantees. In 58th IEEE Conference on Decision and Control. IEEE, 2019.

[3] F. Chen and D. V. Dimarogonas. Second order consensus for leaderfollower multi-agent systems with prescribed performance. IFACPapersOnLine, 52(20):103-108, 2019.

[4] D. V. Dimarogonas and K. H. Johansson. Stability analysis for multiagent systems using the incidence matrix: quantized communication and formation control. Automatica, 46(4):695-700, 2010.

[5] K. Fitch and N. E. Leonard. Optimal leader selection for controllability and robustness in multi-agent networks. In 2016 European Control Conference (ECC), pages 1550-1555. IEEE, 2016.
[6] A. Ilchmann, E. P. Ryan, and S. Trenn. Tracking control: Performance funnels and prescribed transient behaviour. Systems \& Control Letters, 54(7):655-670, 2005

[7] M. Ji, A. Muhammad, and M. Egerstedt. Leader-based multi-agent coordination: Controllability and optimal control. In American Control Conference, 2006, pages 6-pp. IEEE, 2006.

[8] Y. Karayiannidis, D. V. Dimarogonas, and D. Kragic. Multi-agent average consensus control with prescribed performance guarantees. In Decision and Control (CDC), 2012 IEEE 51st Annual Conference on, pages 2219-2225. IEEE, 2012.

[9] I. Katsoukis and G. A. Rovithakis. Output feedback leader-follower with prescribed performance guarantees for a class of unknown nonlinear multi-agent systems. In 2016 24th Mediterranean Conference on Control and Automation (MED), pages 1077-1082. IEEE, 2016.

[10] M. Mesbahi and M. Egerstedt. Graph theoretic methods in multiagent networks, volume 33. Princeton University Press, 2010.

[11] K.-K. Oh, M.-C. Park, and H.-S. Ahn. A survey of multi-agent formation control. Automatica, 53:424-440, 2015.

[12] R. Olfati-Saber and R. M. Murray. Consensus problems in networks of agents with switching topology and time-delays. IEEE Transactions on automatic control, 49(9):1520-1533, 2004.

[13] W. Ren and E. Atkins. Distributed multi-vehicle coordinated control via local information exchange. International Journal of Robust and Nonlinear Control: IFAC-Affiliated Journal, 17(10-11):1002-1033, 2007.

[14] A. Y. Yazicioğlu and M. Egerstedt. Leader selection and network assembly for controllability of leader-follower networks. In American Control Conference (ACC), 2013, pages 3802-3807. IEEE, 2013.

[15] D. Zelazo and M. Mesbahi. Edge agreement: Graph-theoretic performance bounds and passivity analysis. IEEE Transactions on Automatic Control, 56(3):544-555, 2011. 